\title{
La atención primaria de salud en la Región de las Américas a 40 años de la Declaración de Alma-Ata
}

\author{
Gisele Almeida, ${ }^{1}$ Osvaldo Artaza, ${ }^{2}$ Nora Donoso ${ }^{3}$ y Ricardo Fábrega ${ }^{1}$
}

Forma de citar

Almeida G, Artaza O, Donoso N, Fábrega R. La atención primaria de salud en la Región de las Américas a 40 años de Alma-Ata. Rev Panam Salud Publica. 2018;42:e104. https: / / doi.org/10.26633/ RPSP.2018.104

RESUMEN En este artículo se reseña la evolución de las propuestas y acuerdos regionales desde la Declaración de Alma-Ata (1978) hasta la Estrategia de Salud Universal y se destaca la vigencia de los planteamientos esenciales de la estrategia de atención primaria, que hoy se expresan en las propuestas de fortalecimiento del primer nivel de atención y la generación de redes integradas de servicios de salud. Se recuerda, también, el carácter contextual de la implementación de la estrategia en el marco de situaciones nacionales complejas a través de algunos hitos de los últimos 40 años. Se describen los factores que frenan la implementación de la atención primaria de salud (APS), así como los avances y desafíos emergentes que hoy en día enfrentan los sistemas de salud en varios países. Se reafirma que solo mediante un fuerte primer nivel articulador y resolutivo, cercano, inserto en la comunidad y accesible, es posible avanzar hacia el ejercicio del derecho a la salud para todos y se aboga por la generación de propuestas prácticas para relanzar la estrategia de APS a 40 años de la Declaración de Alma-Ata.

Palabras clave Atención primaria de salud; sistemas de salud; política de salud; cobertura universal.

La salud, entendida como un "estado de completo bienestar físico, mental y social, y no solo la ausencia de afecciones o enfermedades" (1), es un fenómeno social complejo que depende de la interacción de múltiples factores sociales, políticos, económicos, culturales y científicos. Dada esta complejidad, no es de extrañar que los líderes de las

\footnotetext{
Organización Panamericana de la Salud, Washington D.C., Estados Unidos de América. Enviar la correspondencia a Gisele Almeida, almeidag@paho.org

Hospital de Valdivia, Bueras, Chile.

Ministerio de Salud, Gobierno de Chile, Chile.
}

naciones reunidos en la Conferencia Internacional de Alma-Ata (Unión de Repúblicas Socialistas Soviéticas, 6 al 12 de setiembre de 1978) (2), hayan propuesto que, para el logro de dicho estado de bienestar y de la materialización del derecho a la salud, la herramienta esencial fuera la atención primaria de salud (APS), que implica una acción integral que va más allá de los enfoques reduccionistas centrados en la enfermedad.

Desafortunadamente, muchos interpretaron a la APS como "servicios pobres para los pobres", nada más alejado al espíritu de Alma-Ata, al punto que algunos protagonistas de la conferencia y de la salud pública regional llegaron a plantear "frustración sobre los malentendidos y las distorsiones que ha sufrido el tema" (3) y señalar directamente "una errada interpretación de los términos" (4). Las condiciones que dieron origen a la meta social y política de "Salud para todos" se fueron profundizando con el tiempo dada la persistencia de grandes inequidades al predominio de la visión médica curativa y a la escasa participación social en los sistemas de salud, lo que explica diversos hitos y

Este es un artículo de acceso abierto distribuido bajo los términos de la licencia Creative Commons Attribution-NonCommercial-NoDerivs 3.0 IGO, que permite su uso, distribución y reproducción en cualquier medio, siempre que el trabajo original se cite de la manera adecuada. No se permiten modificaciones a los artículos ni su uso comercial. Al reproducir un artículo no debe haber ningún indicio de que la OPS o el artículo avalan a una organización o un producto específico. El uso del logo de la OPS no está permitido. Esta leyenda debe conservarse, junto con la URL original del artículo. 
resoluciones (5-9). En setiembre de 2014, el 53. ${ }^{\circ}$ Consejo Directivo de la Organización Panamericana de la Salud (OPS), aprobó la estrategia para el acceso universal a la salud y la cobertura universal de salud, luego abreviadas bajo el lema de Salud Universal, que plantea "que todas las personas y las comunidades tengan acceso, sin discriminación alguna, a servicios integrales de salud, adecuados, oportunos, de calidad..." (10). En la resolución aprobatoria, se señala de manera expresa que "se enmarcan en los valores y principios de la atención primaria de salud, en el espíritu de Alma-Ata", por lo que propone transformar la organización y la gestión de los servicios de salud mediante el desarrollo de modelos de atención centrados en las necesidades de las personas y las comunidades, el aumento de la capacidad resolutiva del primer nivel, articulado en Redes Integradas de Servicios de Salud (RISS) (11) y basado en la atención primaria de salud. Por último, los Objetivos del Desarrollo Sostenible proponen, en su objetivo tercero, garantizar una vida sana y promover el bienestar para todos en todas las edades; para ello, una de sus metas es lograr la cobertura sanitaria universal, en particular la protección contra los riesgos financieros, el acceso a servicios de salud esenciales de calidad y el acceso a medicamentos y vacunas seguros, eficaces, asequibles y de calidad para todos (12), lo que de modo evidente refuerza lo ya planteado desde Alma-Ata (13).

En el discurso y las declaraciones todos parecen estar de acuerdo, pero a la hora de las decisiones, en nuestra opinión, los países siguen privilegiando un modelo centrado en el hospital, la alta tecnología y la curación de enfermedades. A la hora del diseño de políticas, no se prioriza la inversión en infraestructura, recursos humanos y tecnologías y estrategias sociosanitarias para un trabajo efectivo con la comunidad que actúe sobre las determinantes sociales y acerque la salud al hábitat de las personas (14). No obstante, en el contexto del devenir histórico en cada uno de los países (figura 1) a 40 años de la Declaración de Alma-Ata, la APS sigue apareciendo como la clave para materializar el derecho a la salud.

Cabe preguntarse si en esa falta de coherencia entre discurso y acción en la base hay fuertes intereses y valores en juego que privilegian el modelo médico industrial, el que está al servicio de la "salud como negocio" y no a la "salud como derecho". Este modelo, además de perpetuar las determinantes sociales de la salud, hace que los esfuerzos por implementar el espíritu de Alma-Ata parezcan contracorriente.

El contexto histórico y sociopolítico en América Latina ha mostrado diversa preocupación de los estados por la cuestión social, alternancia de gobiernos democráticos y presencia de gobiernos autoritarios, dictaduras militares, procesos de transición y recuperación de las democracias, procesos de ajuste estructural y reformas del Estado, reformas sanitarias, incorporación de modelos de contención de costos y desarrollo de sistemas de protección social, entre otros procesos a veces divergentes. Estos fenómenos sociopolíticos no siempre han favorecido la implementación de la APS (15). Por una parte, algunos modelos económicos han centrado su mirada en la eficiencia en el gasto público en salud y, muchas veces, dejaron en un segundo plano las necesidades y demandas explícitas de las comunidades. En otros casos, relaciones de carácter más bien populista entre los estados y la sociedad civil, han puesto el acento en acciones de carácter cortoplacistas que no logran un impacto real en la salud de la población. Por otro lado, la tendencia a la planificación "desde arriba" ha frenado procesos de descentralización política y administrativa que posibiliten la incorporación de la participación intersectorial en procesos de planificación local con enfoque estratégico y sustentable. A pesar de que distintos procesos dan cuenta de la intencionalidad de promover un cambio en el eje de las acciones de salud desde los puntos de vista individual y asistencial hacia las acciones colectivas, preventivas y de promoción, resultan insuficientes para alterar la práctica tradicional biomédica y asistencial a nivel de la APS (16).

No obstante, a pesar de las dificultades, se pueden constatar avances (cuadro 1). Se observa que las tasas de inmunización en la Región se mantienen elevadas, se aumenta de manera importante la cobertura de tratamiento antirretroviral, se duplica el gasto per cápita en salud y baja la mortalidad infantil, materna y por causas evitables. Las políticas de salud implementadas en las últimas décadas en la Región de las Américas, dirigidas a aumentar la cobertura en salud, se han dado en un contexto de redemocratización y crecimiento económico, lo que permitió un aumento en el financiamiento, reducción de gastos catastróficos, mejores indicadores de salud y reducción de las desigualdades. Estas políticas han aumentado la cobertura en salud para más de 46 millones de latinoamericanos

\section{FIGURA 1. Cronología de hitos: de Alma-Ata a la Salud Universal}

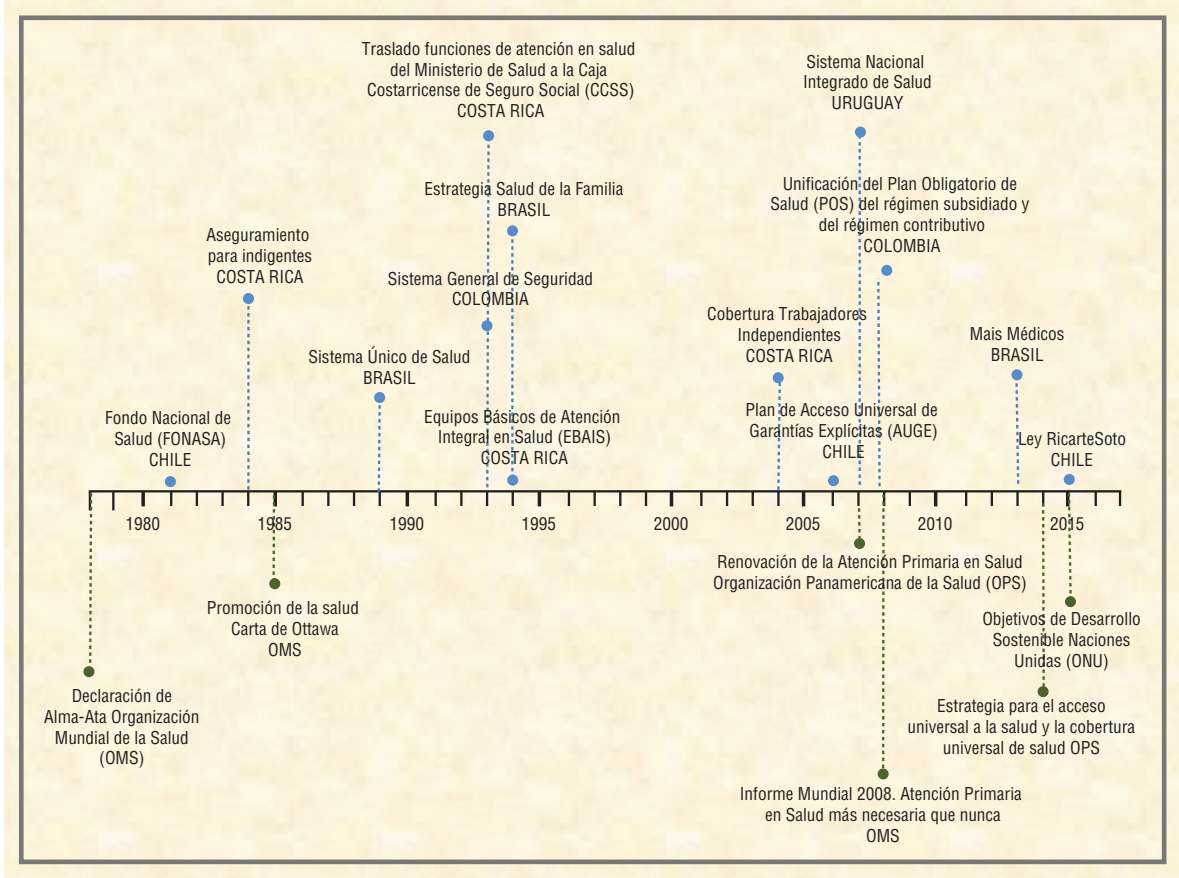


CUADRO 1. Indicadores demografícos, económicos y relacionados con el sistema de salud, factores de riesgo y mortalidad en América Latina y el Caribe, 2000-2015

\begin{tabular}{|c|c|c|c|c|}
\hline Indicadores & 2000 & 2005 & 2010 & 2015 \\
\hline \multicolumn{5}{|l|}{$\overline{\text { Demográficos }}$} \\
\hline Población total & 524829251 & 560677885 & 596479937 & 631062661 \\
\hline Crecimiento de la población (\% anual) & 1,45 & 1,28 & 1,21 & 1,08 \\
\hline Población $\geq 65$ años (hombres, \% del total) & 4,87 & 5,37 & 5,94 & 6,72 \\
\hline Tasa de fecundidad (nacimientos por mujer) & 2,60 & 2,40 & 2,20 & 2,10 \\
\hline Esperanza de vida al nacer (años) & 71,53 & 72,98 & 74,22 & 74,22 \\
\hline \multicolumn{5}{|l|}{ Económicos } \\
\hline Desempleo (\% de la población activa total)a & 10,61 & 8,00 & 7,26 & 6,57 \\
\hline Tasa de incidencia de pobreza (\% de la población total) ${ }^{b}$ & 11,70 & 9,80 & 6,00 & 4,50 \\
\hline PIB per cápita, PPA (dólar internacional actual)` & 8635,1 & 10422,6 & 13280 & 15238,2 \\
\hline Gasto actual en salud ( $\%$ del PIB $)^{d}$ & 6,10 & 6,52 & 6,87 & 7,39 \\
\hline Gasto actual en salud per cápita, PPA (dólar internacional actual) ${ }^{\mathrm{d}}$ & 536,70 & 677,49 & 894,78 & 1081,34 \\
\hline Gasto nacional en salud per cápita, PPA (dólar internacional actual) ${ }^{\text {d }}$ & 236,94 & 300,07 & 439,73 & 556,71 \\
\hline Gastos de bolsillo ( $\%$ del gasto actual en salud) ${ }^{d}$ & 42,07 & 42,36 & 33,95 & 31,27 \\
\hline \multicolumn{5}{|l|}{ Sistema de salud } \\
\hline Inmunización con vacuna DPT ${ }^{\mathrm{e}}$ & 90,73 & 93,61 & 93,21 & 90,09 \\
\hline Inmunización contra el sarampióne & 93,58 & 92,73 & 93,28 & 92,66 \\
\hline Cobertura del tratamiento antirretroviral ${ }^{\dagger}$ & 10,40 & 20,80 & 33,86 & 53,09 \\
\hline \multicolumn{5}{|l|}{ Factores de riesgo } \\
\hline Prevalencia de desnutrición según la altura para la edadg & 18,40 & 15,70 & 13,40 & 11,40 \\
\hline Prevalencia de sobrepeso ${ }^{g}$ & 6,80 & 6,80 & 6,90 & 7,00 \\
\hline Prevalencia de desnutrición (\% de la población total) & 11,95 & 8,99 & 6,93 & 6,58 \\
\hline \multicolumn{5}{|l|}{ Mortalidad } \\
\hline ECV, cáncer, diabetes o ERC (30-70 años de edad, \%) & 20,42 & 28,58 & 17,29 & 15,99 \\
\hline Suicidios (por cada 100000 habitantes) & 6,82 & 6,94 & 6,93 & 7,05 \\
\hline Tasa de mortalidad en $<5$ años (por cada 1000 niños nacidos vivos) & 33,43 & 26,19 & 24,62 & 18,07 \\
\hline Tasa de mortalidad infantil (por cada 1000 niños nacidos vivos) & 27,72 & 21,97 & 18,79 & 15,33 \\
\hline Tasa de mortalidad materna (por cada 100000 nacidos vivlos) & 99,00 & 88,00 & 81,00 & 67,00 \\
\hline
\end{tabular}

aSobre la base del modelo de la Organización Internacional del Trabajo.

'Sobre la base de \$1,90/día (2011 PPA).

'Producto interno bruto (PIB) per cápita basado en la paridad del poder adquisitivo (PPA) es el producto interno bruto convertido a dólares internacionales utilizando tasas de paridad del poder adquisitivo y dividido por la población de mitad de año. Los datos de América Latina y el Caribe se calculan con el promedio ponderado.

'Método de agregación: promedio ponderado.

ePorcentaje de niños de entre 12 y 23 meses de edad.

tPorcentaje de las personas que conviven con el virus de la inmunodeficiencia humana.

${ }^{9}$ Porcentaje de niños menores de cinco años.

ECV,enfermedad cerebrovascular; ERC, enfermedad renal crónica. DPT; difteria, tétanos y tos convulsa.

Fuentes: Indicadores del desarrollo mundial, Banco Mundial (http://databank.bancomundial.org/data/reports.aspx?source=world-development-indicators).

Organización Mundial de la Salud. Global Health Expenditure database (http://apps.who.int/nha/database).

Organización Mundial de la Salud. Global Health Observatory Data Repository (http://apps.who.int/ghodata/).

en los últimos 25 años (17). Los países que han implementado políticas y programas enfocados en la APS registran las menores tasas de mortalidad infantil y materna, como el caso de Chile, Costa Rica y Uruguay (18-20). Estos países también presentan una tendencia positiva en el aumento del uso de servicios de prevención del cáncer de cuello uterino y de seno, del uso de anticonceptivos, de las consultas prenatal y de los partos atendidos por personal calificado (17).

Chile, por ejemplo, desde la creación del Servicio Nacional de Salud en el año 1952, tiene una larga historia de fortalecimiento gradual y constante del primer nivel de atención, en especial en las últimas décadas con la implementación del modelo de salud familiar y comunitaria (21) y un fuerte incremento del financiamiento público (22). Esta trayectoria consolida a la APS como una exitosa política de Estado de amplio consenso. Esto se ve retratado en que diversos actores suscribieron hace poco, en ese país, un documento de posición que recoge opiniones de más de 10000 personas participantes en 387 conversatorios realizados entre los años 2016 y 2017 sobre el fortalecimiento del valor social de la APS $(23,24)$.

En varios países de la Región, ha habido intentos serios de incorporar a la sociedad civil y comunidades locales, en distintos espacios de participación en salud; la creación de instancias formales de representación de organizaciones sociales y de base comunitaria como comités locales de salud o consejos de salud (Bolivia, Brasil, Chile, Colombia, México y Perú, entre otros) con diferentes grados de incidencia en el diseño, implementación, evaluación de las políticas de salud; el ejercicio de rendición de cuentas de las autoridades de salud a través de las cuentas públicas participativas; el ejercicio de presupuestos participativos como espacios deliberativos y de incidencia en el uso de recursos públicos en salud, y distintas formas de 
diálogo y consultas ciudadanas (presenciales o a través de plataformas digitales participativas) en relación a modificaciones de carácter legal (etiquetado de los alimentos, derechos y garantías explícitas en salud, producción y distribución de fármacos) o transformaciones tecnológicas que pueden afectar la salud de la población $(25,26)$.

Uno de los grandes desafíos identificados para los sistemas de salud, que deberían tener su resolución sobre todo en la atención primaria, es el incremento de las enfermedades crónicas no transmisibles y factores de riegos asociados, que han aumentado en el tiempo en todos los grupos socioeconómicos en todos los países (17). El rol del primer nivel de atención para la reducción de las enfermedades crónicas y factores de riesgos es esencial. La inversión en el aumento de la capacidad del primer nivel de atención para la prevención de enfermedades, diagnóstico temprano y detección de los factores de riesgos es uno de los pilares principales de la Declaración de AlmaAta y de la Estrategia de Salud Universal. Una de las recomendaciones en relación con servicios de salud preventiva para adultos es realizar por lo menos una consulta preventiva por año. Sin embargo, y a pesar del aumento en el tiempo, en varios países de América Latina el promedio anual de consultas preventivas está alrededor de $20 \%$, lo que representa una brecha significativa con respecto a lo que debería ser un modelo de atención preventiva (17). Aún son elevadas las hospitalizaciones por condiciones sensibles al cuidado ambulatorio, así como su costo. Un estudio publicado en 2012 ha estimado el número de hospitalizaciones evitables entre 8,1 y 10 millones en varios países de América Latina; el costo asociado a estas hospitalizaciones representa 2,5\% del gasto total en salud en estos países (27). Estos resultados señalan que, con una mejor capacidad resolutiva en la atención primaria, muchas hospitalizaciones se podrían evitar $y$, de esta manera, reducir el costo y mejorar la calidad de vida de la población.

A la luz de los avances y los aspectos pendientes es necesario reafirmar que APS es la asistencia sanitaria esencial accesible a todos los individuos y familias de la comunidad a través de medios aceptables para ellos, con su plena participación y a un costo asequible para la comunidad y el país. Por esencial se debe entender a lo primordial, lo más importante que debe estar accesible para todos de manera integral, en la múltiple significación de integralidad: 1) fomento, prevención, curación, rehabilitación, paliativo y cuidados de largo plazo, 2) en todo el curso de vida, 3) en el continuo de atención y cuidados, y 4) en la diversidad de ser familias y comunidad, sin discriminación alguna para la obtención del mayor nivel de calidad de vida posible. Por lo tanto, es una estrategia que atraviesa no solo todo el sector salud, sino a todas las políticas. Por ello, es clave un primer nivel de atención fuerte, resolutivo y de calidad que este apoyado por servicios especializados estructurados en red. La estrategia de APS requiere de una activa participación social y de incorporar aspectos tales como interculturalidad y género, con el fortalecimiento de experiencias como las ya señaladas en algunos países de la región. Sin duda, la APS no se trata de "servicios pobres para los pobres", sino que es la mejor inversión para lograr la salud para todos.

Ampliar el acceso a los servicios de salud implica mejorar la accesibilidad y la capacidad resolutiva en el espacio ambulatorio (fuera de los muros del hospital tradicional), que conlleve a mejorar la confianza y satisfacción de las personas y comunidades. Se entiende por servicios ambulatorios al primer nivel de atención o de contacto con la comunidad usuaria y también a los diversos establecimientos de salud de competencias humanas y capacidades tecnológicas especializadas en temas de menor frecuencia relativa y de soporte diagnóstico, terapéutico o logístico al primer nivel, y a los diversos dispositivos en la comunidad tanto del sector salud como sociales, que en un trabajo articulado en red, resuelven -sin internación- las necesidades de personas y comunidades (28).

El abordaje anticipatorio del cuidado de la salud $y$, por ende, la mejora en la calidad de vida, implica una respuesta social integrada. Este cambio no puede ser adscrito solo al sector salud o a un dispositivo sanitario en particular e implica un cambio en el paradigma organizativo, en cómo intervenimos desde el punto de vista poblacional, en cómo se modelan los sistemas y en cómo disponemos los recursos necesarios para cumplir ese objetivo (28).

La capacidad resolutiva de APS incluye contar con equipos interdisciplinarios de salud capacitados y con incentivos y condiciones laborales atractivas, sobre todo en áreas con dificultades en el acceso a la atención en salud, como por ejemplo áreas rurales dispersas o zonas urbanas y periurbanas pobres y excluidas. Dicho personal debe contar con las tecnologías sanitarias apropiadas (laboratorios, medicamentos) y suficientes, así como con el apoyo efectivo de las especialidades. Los cambios en el primer nivel de atención se dirigen hacia tener un equipo de salud que cuente con las capacidades para actuar de manera intersectorial sobre los determinantes sociales de la salud, para actuar junto a la comunidad en acciones de promoción y prevención, para el manejo tanto de los problemas agudos como de los crónicos, incluidas las secuelas y discapacidades, así como los cuidados paliativos, en y desde el propio domicilio de las personas, y para sostener una relación cercana, fluida, con pertinencia y permanente con la comunidad en su contexto territorial. En una lectura actual del espíritu de Alma-Ata, la participación es más que una mera formalidad, es el medio para dar real poder y protagonismo a personas y comunidades. La interculturalidad, más que un enfoque complementario, es un cambio paradigmático que implica la traducción a nuestras prácticas del respeto a la diversidad. La cercanía tiene una dimensión relacionada a la ausencia de barreras de acceso geográficas, económicas, culturales, entre otras, que implica: 1) la proximidad física y la facilidad con que las personas establecen un vínculo con el personal de salud, piden sus citas, reciben información, son involucrados y gozan de la comprensión de sus necesidades particulares sean por edad, género, etnia $u$ otra característica, 2) la vinculación con los diversos actores locales y la realidad territorial, incluidos los medios de comunicación, con el fin de disponer de servicios que forman parte integral de la vida comunitaria: el uso de las tecnologías modernas de la comunicación, la visita domiciliaria, las brigadas o rondas móviles, el agente o trabajador de salud comunitario, las alianzas con grupos de la sociedad civil, y muchas otras modalidades como estrategias para fortalecer estos vínculos con las comunidades locales, y 3) el avance en la generación de procesos de planificación local participativa en marcos crecientes de descentralización político-administrativa, la búsqueda de la equidad y pertinencia cultural de las acciones de salud, la 
transversalización del enfoque de derechos y ejercicio de la ciudadanía activa en el desarrollo de una agenda de inclusión y protección social sostenible (28).

El primer nivel no es solo una puerta de entrada, sino que se constituye en eje ordenador de todo el sistema y en la fuente primaria de los sistemas de información para el manejo y gestión de casos (que se ve apoyado por el desarrollo de expedientes electrónicos en red), para seguimiento de procesos asistenciales, gestión de turnos (citaciones) y casos, y para sistemas de referencia y retorno, entre otros. Esto último

1. Organización Mundial de la Salud. Constitución de la Organización Mundial de la Salud. Disponible en http://www. who.int/governance/eb/who_constitution_sp.pdf Acceso el 4 de enero de 2018.

2. Organización Panamericana de la Salud. Declaración de Alma-Ata. Disponible en: http:/ / www.paho.org/hq/index.php? option=com_docman\&task $=$ doc_view \& gid $=19004 \&$ Itemid $=270 \& l a n g=e n$ Acceso el 4 de enero de 2018.

3. Tejada de Rivero DA. Alma-Ata: 25 años después. Revista Perspectivas de Salud. La revista de la Organización Panamericana de la Salud. 2003;8(2):3-7. Disponible en: http://cidbimena.desastres.hn/docum/ ops/Revistas/persp17span.pdf

4. Tejada de Rivero DA. Lo que es la atención primaria de la salud: algunas consideraciones a casi treinta y cinco años de AlmaAta. Rev Peru Med Exp Salud Publica. 2013;30(2):283-7. 30. Disponible en: http:/ / www.scielosp.org/scielo.php?script $=$ sci_arttext\&pid=S1726-4634201300020 0020\&lng=en Acceso el 4 de enero de 2018.

5. Organización Panamericana de la Salud (OPS). $44 .{ }^{\circ}$ Consejo Directivo. Atención primaria de salud en las Américas: las enseñanzas extraídas a lo largo de 25 años y los retos futuros. Washington, D.C.: OPS; 2003. Disponible en: http://www1.paho. org/spanish/gov/cd/.cd44-r6-s.pdf Acceso el 4 de enero de 2018.

6. Macinko J, Montenegro H, Nebot Adell C, Etienne C y Grupo de Trabajo de Atención Primaria de Salud de la Organización Panamericana de la Salud. La renovación de la atención primaria de salud en las Américas. Rev Panam Salud Publica. 2007;21(2/3):73-84. Disponible en: http:/ / www.scielosp.org/pdf/rpsp/v21n2-3/03. pdf Acceso el 4 de enero de 2018.

7. Periago MR. La renovación de la atención primaria de salud en las américas: la propuesta de la organización panamericana de la salud para el siglo XXI. Rev Panam Salud Publica. 2007;21(2-3):65-8. Disponible en: http:/ / www.scielosp.org/scielo.php? script $=$ sci_arttext $\&$ pid $=S 1020-49892$ 007000200001\&lng=en Acceso el 4 de enero de 2018. significa que los recursos humanos, infraestructura y tecnología de los servicios se estructuran y organizan para asegurar un primer nivel capaz de actuar con y para las personas y la comunidad. Esto permite la resolución integral de los principales problemas de salud desde el propio hábitat de las personas.

Por todo lo señalado, transcurridos 40 años desde la Declaración de Alma-Ata, este resulta un buen momento para reflexionar no solo sobre un diagnóstico de situación, sino para proponer soluciones concretas y prácticas nacidas del diálogo,

\section{REFERENCIAS}

8. Organización Mundial de la Salud (OMS). La atención primaria de salud más necesaria que nunca. Informe sobre la salud en el mundo 2008. Ginebra: OMS; 2008. Disponible en: http:/ / www.who.int/whr/ 2008/08_report_es.pdf Acceso el 4 de enero de 2018.

9. Naciones Unidas. Resolución aprobada por la Asamblea General 67/81. Salud mundial y política exterior, A/RES/67/81 (14 de marzo de 2013). Disponible en: http://www.un.org/es/comun/docs/?symbol=A/RES/67/81 Acceso el 4 de enero de 2018

10. Organización Panamericana de la Salud (OPS). Consejo Directivo 53/5. Estrategia para el acceso universal a la salud y la cobertura universal de salud. Washington D.C.: OPS; 2014. Disponible en: http:// www.paho.org/uhexchange/index.php/ es/uhexchange-documents/informaciontecnica/27-estrategia-para-el-acceso-universal-a-la-salud-y-la-cobertura-universalde-salud/file Acceso el 4 de enero de 2018.

11. Organización Panamericana de la Salud (OPS). Redes integradas de servicios de salud: conceptos, opciones de política y hoja de ruta para su implementación en las Américas. Washington D.C.; OPS; 2010. Disponible en: http://www.paho. org / uru/index.php?option=com_docman\&view=download\&category_slug= publicaciones-sistemas-y-servicios-de-salud\&alias $=145$-redes-integradas-de-servicios-de-salud-aps-n4\&Itemid=307 Acceso el 4 de enero de 2018.

12. Transformar nuestro mundo: la Agenda 2030 para el Desarrollo Sostenible. Resolución aprobada por la Asamblea General el 25 de setiembre de 2015. Disponible en: http://www.un.org/es/comun/docs/? symbol=A/RES/70/1 Acceso el 4 de enero de 2018.

13. Organización de las Naciones Unidas. Objetivos de Desarrollo Sostenible. Disponible en: https://www.un.org/sustainabledevelopment/es / objetivos-dedesarrollo-sostenible/ Acceso el 4 de enero de 2018.

14. Artaza O, Sagastuy B. De Salud para Todos a Salud Universal: cómo concretar el derecho a la protección a la salud. Salud la escucha y la investigación rigurosa. Si no hay atención primaria fortalecida, con participación intersectorial y de las comunidades, no habrá bienestar social ni acceso universal a la salud.

Conflicto de intereses. Ninguno declarado por los autores.

Declaración. Las opiniones expresadas en este manuscrito son responsabilidad del autor y no reflejan necesariamente los criterios ni la política de la RPSP/ PAJPH y/o de la OPS.

Problema. 2017;11:47-66. Disponible en https:/ / publicaciones.xoc.uam.mx Acceso el 4 de enero de 2018.

15. Greer SL, Méndez CA. Universal health coverage: a political struggle and governance challenge. Am J Public Health. 2015;105: S637-S639.

16. Cunill Grau N, Fernández MM, Vergara M. Gobernanza sistémica para un enfoque de derechos en salud: Un análisis a partir del caso chileno. Salud Colectiva. [Internet]. 2011(1):21-33. Disponible en: http:/ / www. scielo.org.ar/scielo.php?script=sci_arttext\&pid=S1851-82652011000100003\&lng=es Acceso el 4 de enero de 2018.

17. Dmytraczenko T, Almeida G. Hacia la cobertura universal en salud y la equidad en América Latina y el Caribe: evidencia de países seleccionados. Banco Mundial. Directions in DevelopmentHuman Development: 2015. Disponible en: https: / / openknowledge.worldbank. org/bitstream/handle/10986/22026/ 9781464811777.pdf? sequence $=8 \&$ isAllowed=y Acceso el 4 de enero de 2018.

18. Sáenz MR, Acosta M, Muiser J, Bermúdez JL. Sistema de salud de Costa Rica. Salud Publica Mex. 2011;53(S2):s156-s167. Disponible en: http://www.scielo.org. $\mathrm{mx} /$ scielo.php?script=sci_arttext\&pid= S0036-36342011000800011\&lng=es Acceso el 4 de enero de 2018.

19. Téllez A. Atención primaria: factor clave en la reforma al sistema de salud. Santiago de Chile: Pontificia Universidad Católica de Chile; 2015. Disponible en: http:/ / politicaspublicas.uc.cl/wp-content/uploads / 2015/02/atencion-primaria-factor-claveen-la-reforma-al-sistema-de-salud.pdf Acceso el 4 de enero de 2018.

20. Organización Panamericana de la Salud (OPS). Atención Primaria de Salud en Uruguay: experiencias y lecciones aprendidas. Montevideo: OPS; 2011. Disponible en https://uruguay.campusvirtualsp.org/ sites/default/files/webfiles/contenido/ OPS $\% 20-\% 20$ libro $\% 20 \mathrm{de} \% 20$ APS-2011FINAL-tapas.pdf Acceso el 4 de enero de 2018.

21. Ministerio deSalud de Chile. Orientaciones para la implementación del Modelo de Atención Integral de Salud familiar y 
comunitaria. Santiago de Chile: Ministerio de Salud; 2013 Disponible en http://web. minsal.cl/portal/url/item/e7b24eef 3e5cb5d1e0400101650128e9.pdf Acceso el 4 de enero de 2018.

22. Pontificia Universidad Católica de Chile. Centro De Políticas Públicas UC. Fortalecimiento de la atención primaria de salud: propuestas para mejorar el sistema sanitario chileno. 2014;9(67). Disponible en http://politicaspublicas.uc.cl/wp-content/ uploads / 2015/02/fortalecimiento-dela-atencion-primaria-de-salud-propuestaspara-mejorar-el-sistema-sanitario.pdf Acceso el 4 de enero de 2018.

23. Ministerio de Salud de Chile. Documento de posición respecto del valor social de la atención primaria de salud. Santiago de Chile: Ministerio de Salud; 2017. Disponible en http://web.minsal.cl/wpcontent/uploads / 2016/11/Posici $\%$ C3\% B3n-respecto-del-valor-social-APS Acceso el 4 de enero de 2018
24. Ministerio de Salud de Chile. Norma General de Participación Ciudadana en la Gestión Pública de Salud. Santiago de Chile: Ministerio de Salud; 2015.

25. Donoso Valenzuela, N. Los presupuestos participativos en salud. En: Participación Social en Salud: Reflexiones y Herramientas para la Acción desde Chile. Santiago de Chile: Ministerio de Salud; 2009: 91-102. Disponible en: http://www.bibliotecaminsal.cl/wp/wp-content/uploads / 2016/03/Participacion-Social-en-Salud. pdf Acceso el 4 de enero de 2018.

26. Donoso Valenzuela, N. Participación y cohesión social como ejes de la reforma y del modelo de atención en salud. En: Participación Social en Salud: Reflexiones y Herramientas para la Acción desde Chile. Santiago de Chile: Ministerio de Salud; 2009:11-20. Disponible en http://www. bibliotecaminsal.cl/wp/wp-content/ uploads/2016/03/Participacion-Social-enSalud.pdf Accesoe 14 de enero de 2018.
27. Guanais FC, Gómez-Suárez R, Pinzón L. Primary care effectiveness and the extent of avoidable hospitalizations in Latin America and the Caribbean. En: Series of Avoidable Hospitalizations and strengthening Primary Health Care. Washington, D.C.: Banco Interamericano de Desarrollo: 2012.

28. Organización Panamericana de la Salud (OPS). Transformando los servicios hacia redes integradas. Buenos Aires: OPS; 2017. Disponible en: http://publicaciones.ops. org.ar/publicaciones/coleccionOPS/ pub/pub71.pdf Acceso el 4 de enero de 2018

Manuscrito recibido el 12 de enero de 2018. Aceptado para su publicación, tras revisión, el 22 de mayo de 2018.

ABSTRACT This article reviews the evolution of regional proposals and agreements from the Declaration of Alma-Ata (1978) to the Universal Health Strategy, highlighting how the core tenets of the primary health care strategy have come to be reflected in proposals to strengthen the primary level of care and establish integrated health services networks. Contextual aspects of implementing the strategy within the framework of complex national scenarios are also noted, through a review of some of the milestones of the last 40 years. Factors that hinder implementation of primary health care are described, as well as the advances and the emerging challenges that health systems face in several countries. This article reaffirms the need for a strong primary care level-with coordination and response capacity, close to and involved in the community, and accessible - in order to advance towards realizing the right to health for everyone. It also advocates for practical proposals to relaunch the primary health care strategy 40 years after the Declaration of Alma-Ata.

Keywords Primary health care; health systems; health policy; universal coverage.

RESUMO Este artigo apresenta a evolução das propostas e acordos regionais a partir da Declaração de Alma-Ata (1978) até a Estratégia de saúde universal, destacando a vigência das perspectivas básicas da estratégia de atenção primária, atualmente expressas nas propostas de fortalecimento da atenção primária e formação de redes

\section{Atenção primária à saúde na Região das Américas 40 anos após a Declaração de Alma-Ata} integradas de serviços de saúde. Salienta-se o caráter contextual da implementação da estratégia em cenários nacionais complexos, ilustrando-se com os marcos alcançados nos últimos 40 anos. São descritos os fatores que freiam a implementação da atenção primária à saúde (APS) e os avanços e desafios emergentes atualmente enfrentados pelos sistemas de saúde em vários países. Enfatiza-se que, somente com um nível de atenção primário que seja forte, articulado e resolutivo, que esteja próximo e inserido na comunidade e de fácil acesso às pessoas, é possível progredir no direito à saúde para todos. $\mathrm{O}$ artigo defende a elaboração de propostas práticas para relançar a estratégia de APS após 40 anos da Declaração de Alma-Ata.

Palavras-chave

Atenção primária à saúde; sistemas de saúde; política de saúde; cobertura universal. 